\title{
Optical Absorption of Some Glycine Crystals and their Aqueous Solutions
}

\author{
M. KORALEWSKI
}

Institute of Physics, A. Mickiewicz University

Umultowska 85, 61-614 Poznań, Poland

(Received July 1, 2002; revised version December 16, 2002; in final form March 10, 2003)

UV absorption spectra of glycine crystals, i.e. triglycine sulphate, selenate, diglycine sulphate as well as their deuterated analogues in solid state and aqueous solutions are given. Results are compared with the electronic transition energies calculated by LCAO INDO method for glycine molecule and ion, assuming their crystal geometry.

PACS numbers: 78.20.-e, 78.40.-q

\section{Introduction}

Crystals from the triglycine sulphate (TGS) family belong to the most comprehensively studied ferroelectric materials [1, 2]. In recent years the interest in this group of crystals has increased because of a wide range of their applications [3], as e.g. medical vidicons and detectors in infrared spectrometers. Recently, the optical activity (OA) [4], electrooptic effect [5], and birefringence [6, 7] of TGS type crystals in a visible region of the spectrum were discussed, also the application of doped TGS crystals as memory device based on optical gratings has been proposed [8].

TGS crystals are optically transparent in the wavelength range from 0.24 to $1.5 \mu \mathrm{m}$ and their UV optical absorption edge lies near $5 \mathrm{eV}$ [9-11]. The temperature dependence of the UV optical absorption edge was studied by several authors $[9,12,13]$ and the energy gap $\left(E_{\mathrm{g}}\right)$ has been well established for TGS, triglycine selenate (TGSe), and triglycine fluoroberylate (TGFB) crystals. The energy gap slightly decreases with increasing temperature and a change in the slope at the phase transition is observed [9, 12, 13]. Very recently Abu El-Fadl [14] reported a temperature dependence of $E_{\mathrm{g}}$ for pure and $\mathrm{Cu}^{2+}$ doped TGS crystals. 
His results, however, being in good numerical agreement with literature data show a jump-like behaviour at the phase transition in contrast to the results published by others $[9,12,13]$.

The oblique reflection spectra of TGS and TGFB crystals in the range from 5 to $20 \mathrm{eV}$ were reported [15]. The spectra of both crystals are similar and reveal broad bands with a clear structure at a longwave edge. The first maximum is observed near $6.5 \mathrm{eV}$ but a change in the slope can be noticed near $6 \mathrm{eV}$ [15]. Unfortunately, there is no band structure calculation for these crystals, so any assignment or interpretation of the observed bands has not been made.

The absorption bands of the metal doped TGS crystals $[10,16,17]$ in the visible region are easier to interpret because they have been already assigned to the electronic transitions in these metal ions, e.g., $\mathrm{Cu}^{2+}[10,14], \mathrm{Fe}^{3+}[16], \mathrm{Mn}^{2+}[17]$.

As follows from IR and Raman spectroscopy [18] glycine (Gly) and $\mathrm{XO}_{4}^{2-}$ $(\mathrm{X}=\mathrm{S}, \mathrm{Se})$ ions preserve, at least in part their individual properties. Therefore, we may expect the crystals studied to show certain features characteristic of molecular crystals.

No available detailed analysis of the electronic spectra of the TGS group crystals has prompted us to undertake a study of the optical absorption of these crystals and their aqueous solutions in the $\mathrm{UV}$ range.

\section{Experiment and calculations}

Measurements of optical absorption and diffuse reflectance (DR) spectra were carried out in the spectral range from 195 to $900 \mathrm{~nm}$ at room temperature using a Specord M40 spectrophotometer (Zeiss). In the near infrared region a Specord 61 NIR spectrophotometer (Zeiss) was used. Non-polarised light was used. The DR spectra were recorded with the diffuse reflectance attachment, in the geometry $45^{\circ} / 0^{\circ}$, where the first angle is that of light incidence on the sample and the second is that of the reflected light recording. The method of accumulation of the spectra was used and the final result was approximated on the basis of 30 spectra. As a reference standard a powdered pure LiF single crystal was used. The DR spectra are expressed through the remission Kubelka-Munk function [19-21]:

$$
F \equiv \frac{\left(1-R_{\mathrm{D}}^{\infty}\right)^{2}}{2 R_{\mathrm{D}}^{\infty}}=\frac{k}{S},
$$

where $R_{\mathrm{D}}^{\infty}$ stands for diffuse reflectance for such a layer for which an increase in its thickness would not change its remission, $k$ is the absorption coefficient and $S$ is the scattering coefficient of a powdered substance. The function $F$ iis a measure of absorption of the studied material and well reflects the absorption spectrum. The details about DR spectroscopy can be found in our previous paper devoted to alkaline halates [22].

Commercial compounds were recrystallised 5 times and the crystals were grown from aqueous solution by a slow evaporation method. Deuterated crystals 
were grown from a saturated heavy water solution of the studied compound. For the transmission and optical absorption edge studies thin slices of single crystals were cut out in perpendicular to the $Y$ axis and polished to optical quality. For DR studies, the single crystals were grounded to homogeneous fine powder (grain diameter not exceed $2 \mu \mathrm{m}$ ). The aqueous solutions were prepared from single crystals.

The calculations of the electronic transition energies for glycine (molecule, ion) were carried out assuming the geometry they had in the crystal. Numerical calculations were performed by the SCF CI LCAO MO method in INDO approximation making use of GRINDOL programme [23]. The details of the applied modification of INDO method can be found in the papers of Lipiński et al. [24, 25]. As the basis set we used the $1 s$ orbitals of hydrogen atom, $2 s, 2 p$ orbitals of carbon atom and oxygen atom. In the calculations of electronic transition energies, 60 single excitations from the ground state in the configuration-interaction (CI) were taken into account.

\section{Results and discussion}

For the ferroelectric glycine crystals, the short- and long-wavelength edges of optical absorption were determined, Fig. 1. The long-wavelength optical absorption edge is in the near infrared range at $\sim 1.5 \mu \mathrm{m}$, while the short-wavelength one is below $\sim 240 \mathrm{~nm}$. For selenates a small batochromic shift of the short-wavelength absorption edge is observed, see Fig. 2. This shift follows from the fact that the $\mathrm{SeO}_{4}^{2-}$ group shows a much stronger absorption batochromically shifted relatively to that of the $\mathrm{SO}_{4}^{2-}$ group, which was found comparing the absorptions of the $0.01 \mathrm{~N}$ aqueous solutions of sulfuric and selenic acids. The long-wavelength absorption edge was not shifted. The strong IR band below $5000 \mathrm{~cm}^{-1}$ in the range of $1.5-2 \mu \mathrm{m}$ can be assigned to the vibrations of the $\mathrm{NH}_{3}^{+}$group [26, 27]. This band is practically the same for the sulphate and selenate crystals, but for the deuterated crystals the long-wavelength edge was somewhat shifted towards smaller energies (see Fig. 1).

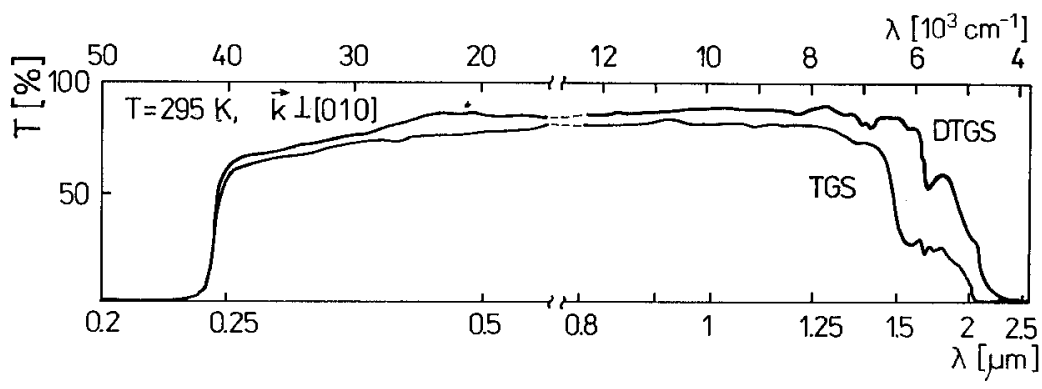

Fig. 1. Transmission spectrum of TGS (thickness $l=1.3 \mathrm{~mm}$ ) and DTGS (thickness $l=1.9 \mathrm{~mm}$ ) single crystals; $k \perp Y, T=295 \mathrm{~K}$ (note the change of scale in IR region). 
It is practically impossible to perform absorption measurements revealing the structure of the spectrum below the short-wavelength absorption edge, because of the problems with obtaining very thin crystal plates of optical quality. This barrier has been partly circumvented by applying the diffuse reflectance spectroscopy in the UV range. The results for powdered crystals of TGS, deuterated triglycine sulphate (DTGS), TGSe, deuterated triglycine selenate (DTGSe), and diglycine sulphate (DGS) are shown in Fig. 2. The same figure presents the positions of the optical absorption edges of the monocrystals of TGS and TGSe, which are consistent with the earlier studies of Hilczer and Balanicka [10] and Abu El-Fadl [14] for TGS, Abass et al. [11] for TGSe. The analysis of the diffusion reflectance spectrum and the edges of optical absorption has shown that the weak absorption band at $\lambda \approx 208 \mathrm{~nm}\left(48200 \mathrm{~cm}^{-1}, \sim 6 \mathrm{eV}\right)$, observed for TGS, DTGS, and DGS, is the longest wavelength band in the electronic spectrum of crystals containing glycine cations, Fig. 2. In the spectra of TGSe and DTGSe the band at $\lambda \approx 208 \mathrm{~nm}$ is overlapped by strong UV bands, like the one assigned to the $\mathrm{SeO}_{4}^{2-}$ anion, which is batochromically shifted relatively to that corresponding to $\mathrm{SO}_{4}^{2-}$. The position of the band at $\lambda \approx 208 \mathrm{~nm}$ is in very good agreement with the change in the slope near $6 \mathrm{eV}$ of the strong band with a maximum at $\sim 6.5 \mathrm{eV}$ observed in the oblique reflection spectrum [15]. Deuterisation of TGS type crystals practically does not change the short-wavelength absorption edge, but a small increase in intensity of the band at $\lambda \approx 208 \mathrm{~nm}$ is observed.

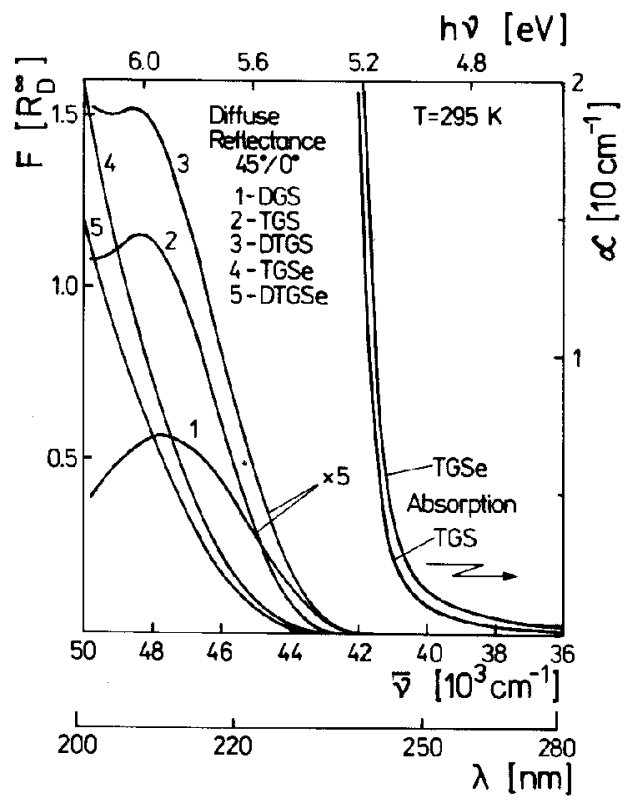

Fig. 2. Diffuse reflectance spectra of some powdered glycine crystals and optical absorption edge of the TGS and TGSe single crystals ( $\boldsymbol{k} \perp Y, T=295 \mathrm{~K}$ ). 


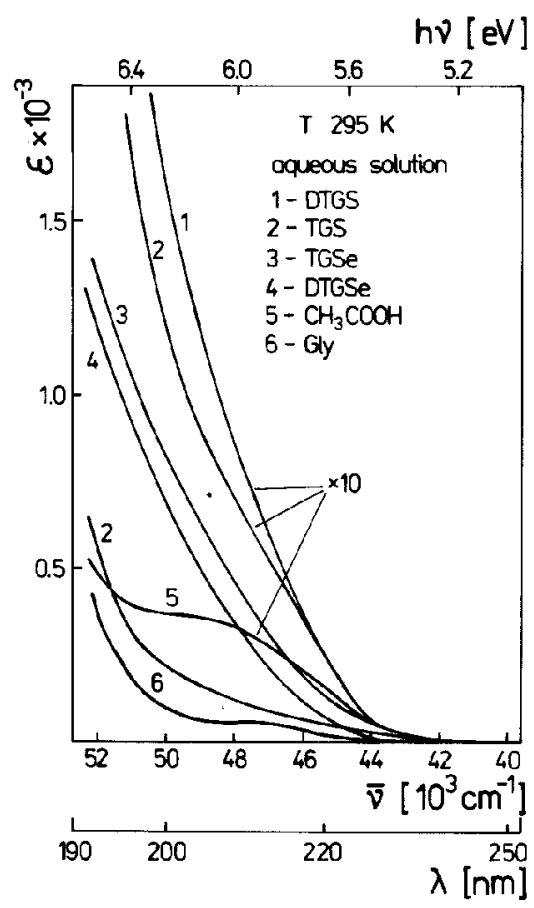

Fig. 3. Spectral dependences of absorption coefficient $\varepsilon$ of aqueous solution of glycine, glycine crystals, and acetic acid $(T=295 \mathrm{~K})$.

The absorption spectra of aqueous solutions of TGS, DTGS, TGSe, DTGSe, and Gly were measured (Fig. 3). The spectra of the aqueous solutions of TGS and DTGS reveal weak inflection points at about $\lambda \approx 206 \mathrm{~nm}\left(48500 \mathrm{~cm}^{-1}, \sim 6 \mathrm{eV}\right)$, despite a strong overlapping effect by the bands below $180 \mathrm{~nm}$. In the spectra of the TGSe and DTGSe solutions the band at $\lambda \approx 206$ is much stronger overlapped by the bands assigned to $\mathrm{SeO}_{4}^{-2}$, however, we can infer its presence on the basis of the shape of the broad wings observed. For glycine (Gly) aqueous solution a very weak diffuse band with a maximum around $208 \mathrm{~nm}$ merging into continuous absorption may be seen. At this point it is worth noting that Gly is the subject of many studies, however they are mainly related to IR and Raman spectra (e.g. [28]) or to Gly ionicity changes in aqueous solution [29]. We were able to cover a small spectral range with a rather poor resolution, a discussion of the above-mentioned problems is left for independent future study. Additional optical measurements were performed for aqueous solutions of alanine (Ala) and acetic acid, i.e. compounds with the same optical chromophore as Gly. The spectrum of aqueous solution of acetic acid (Fig. 3) reveals a weak but distinct band at $\lambda \approx 208 \mathrm{~nm}\left(48000 \mathrm{~cm}^{-1}, \sim 6 \mathrm{eV}\right)$. This weak band which extends from 200-240 nm was assiqned [30-32] to the $n-\pi^{*}$ transition in carboxyl group as 


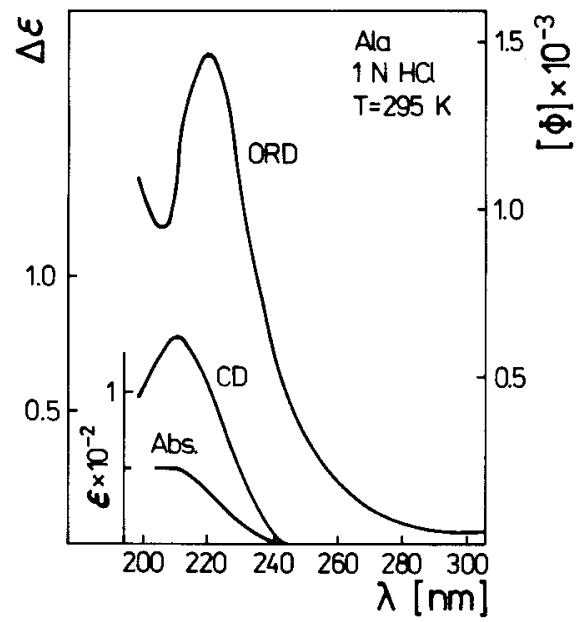

Fig. 4. The optical absorption, ORD, and CD spectra of aqueous solution of alanine.

an obvious optical chromophore of acetic acid. The results of additional measurements of absorption, optical rotatory dispersion (ORD) and circular dichroism (CD) of an aqueous solution of Ala are presented in Fig. 4. The absorption band at $\lambda \approx 210 \mathrm{~nm}$ in the alanine spectrum is much stronger than in the glycine spectrum, see Fig. 3. This absorption band is related to the CD and ORD curves of the positive Cotton effect. The band at $\lambda \approx 210 \mathrm{~nm}$ observed in ORD appears on the slope of a much stronger band or bands in far ultraviolet (Fig. 4) characterised by a great rotatory power $R_{\mathrm{ab}}$. The structure of Ala shows that except for the carboxyl group it has no obvious chromophores. It is well established $[33,34]$ that an absorption band at about $\lambda \approx 210 \mathrm{~nm}$, which is common for all natural amino acids, is resulting from $n-\pi^{*}$ transition in carboxyl group. Also it is known that the position and intensity of $n-\pi^{*}$ transition depend on the $\mathrm{pH}$ of the solution $[33,34]$ which may explain small changes observed in the solutions studied. It is worth noticing that the calculation of rotatory power of the $210 \mathrm{~nm}$ band $\left(R_{210}\right)$ of Ala gives the result $R_{210}=2.4 \times 10^{-40}$ [cgs], which is in good agreement with the model theoretical calculation of the rotatory power for $n-\pi^{*}$ transition in carboxyl chromophore of lactic acid [35].

Because glycine, due to the lack of the chiral centre, does not show optical activity, the spectra ORD and CD cannot be used to help to interpret the weak absorption band at $\lambda \approx 208 \mathrm{~nm}$.

The INDO method, described in Sec. 2, was used to calculate the electronic excitation energies for individual molecule and ion $\left(\mathrm{G}^{+}\right)$of glycine with an assumption that its geometry is the same as the geometry of $\left(\mathrm{G}^{+}\right)$in TGS and diglycine selenate (DGSe) at room temperature. The $x, y, z$ coordinates of constituent atoms of Gly I and Gly II were taken after paper [36] for TGS and after Olejnik [37] for DGSe crystals. The planar scheme and atomic numbering scheme 

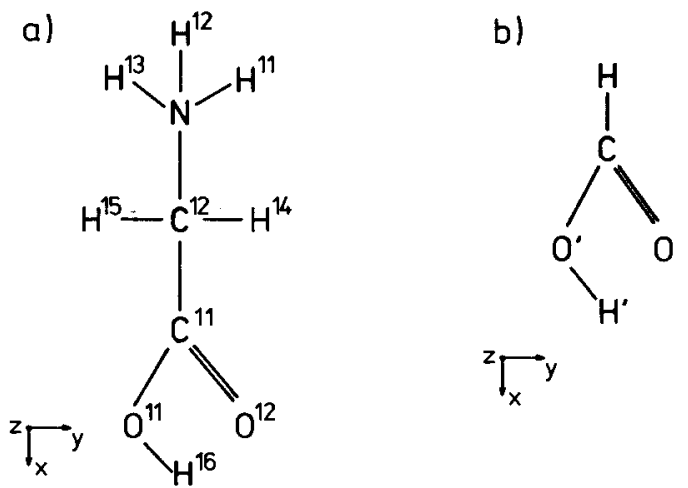

Fig. 5. Planar scheme and atomic numbering of Gly I ion of TGS crystal after Fletcher at al. [36] (a), and $\mathrm{HCOOH}$ molecule after paper [38] (b).

for Gly I of TGS crystal after Fletcher et al. [36] is given in Fig. 5. In the same figure the planar schedule of formic acid after the paper [38] is also given. It is well known [36] that in the TGS crystals Gly II and Gly III are essentially planar and form a positive ion and a zwitterion, respectively but Gly I with the nitrogen atom strongly out-of plane is not planar and form a positive ion, i.e. $\mathrm{NH}_{3}^{\oplus} \mathrm{CH}_{2} \mathrm{COOH}$ and may be described by the symmetry $C_{1}$ only. This fact implies that in the group theory notation only type $a$ orbitals can occur in such a structure of Gly, while in the molecule of $\mathrm{HCOOH}$ of $C_{s}$ symmetry in the ideal structure, both $a^{\prime}$ and $a^{\prime \prime}$ can occur. Within the $C_{1}$ symmetry all transitions are allowed and they all have a symmetry of ${ }^{1} A \leftarrow{ }^{1} A$, however the symmetry of chromophore may play a dominating role in a particular transition and this may explain the intensity of the observed absorption band.

For the sake of comparison similar calculations have been performed for the molecules of alanine for the reason mentioned early (geometry taken after [39]) and formic acid, $\mathrm{HCOOH}$, which is an archetype of compounds with the carboxyl chromophore (geometry taken after [38]). It is worth noting that formic acid has been very recently [40] analysed to show a fine vibrational structure in the $n-\pi^{*}$ transition which extends from 260 to $200 \mathrm{~nm}[40,41]$.

After performed calculations of the ground state of $\mathrm{HCOOH}$ molecule, the following electron filling scheme may be written (notation as in Fig. 5 was used):

$$
\begin{gathered}
\mathrm{O}^{\prime}(2 s)^{2} \mathrm{O}(2 s)^{2} \mathrm{C}(2 s)^{2} \mathrm{O}^{\prime}\left(2 p_{x}\right)^{2} \mathrm{O}^{\prime}\left(2 p_{y}\right)^{2} \mathrm{O}^{\prime}\left(2 p_{z}\right)^{2} \mathrm{O}\left(2 p_{y}\right)^{2} \mathrm{O}\left(2 p_{z}\right)^{2} \mathrm{O}\left(2 p_{x}\right)^{2} \\
\mathrm{C}\left(2 p_{z}\right)^{0} \mathrm{C}(2 s)^{0} \mathrm{H}^{\prime}(1 s)^{0} \mathrm{C}\left(2 p_{x}\right)^{0} \mathrm{C}\left(2 p_{y}\right)^{0}
\end{gathered}
$$

It should be mentioned that atomic orbitals $(\mathrm{AO})$ with the highest linear coefficient which contribute to particular filled and unfilled molecular orbitals (MO) are given in the filling scheme only. Further inspection of AO contributing to the particular MO of the ground state leads to the following scheme (2) using the classical $n$, $\pi, \sigma$ notation as: 


$$
\ldots\left(\sigma_{\mathrm{CO}}\right)^{2}\left(\pi_{\mathrm{CO}}\right)^{2}\left(n_{\mathrm{O}}\right)^{2}\left(\pi_{\mathrm{CO}}^{*}\right)^{0}\left(\sigma_{\mathrm{CH}}^{*}\right)^{0} \ldots .
$$

Calculation of excitation energies within CI treatment shows that some overlapping of several electron configurations in a particular electron excitation exists. The contribution of the electron excitation from $9 \mathrm{MO}$ to $10 \mathrm{MO}$ (from the highest filled orbital to the first unfilled orbital in scheme (2)) to the lowest singlet electronic transition (i.e. $E=5.13 \mathrm{eV}$ - see Table II) reaches $88 \%$, the contributions of the excitation from 7 to $10 \mathrm{MO}$ and 5 to $10 \mathrm{MO}$ are of $4 \%$, while the other excitations bring the remaining contribution. On this ground the main contribution of the AO to the lowest singlet electronic transition can be established as: $\mathrm{O}\left(2 p_{x}\right) \rightarrow \mathrm{C}\left(2 p_{z}\right)$. It may be argued that this transition belongs to the $n-\pi^{*}$ group. Similar results for the lowest singlet-singlet electronic transition were obtained from the calculation performed for $\mathrm{HCOO}^{-}$ion with geometry it has in Gly I of TGS crystal [36], i.e. $\mathrm{O}^{12}\left(2 p_{x}\right) \rightarrow \mathrm{C}^{11}\left(2 p_{z}\right)$ (see notation in Fig. 5, correlation in atom numbering is following: $\left.\mathrm{O}^{\prime}-\mathrm{O}^{11}, \mathrm{H}^{\prime}-\mathrm{H}^{16}, \mathrm{O}-\mathrm{O}^{12}, \mathrm{C}-\mathrm{C}^{11}\right)$. If we take into account the whole positive Gly I ion $\left(\mathrm{G}^{+}\right)$of TGS crystal and the assumption as in discussion of $\mathrm{HCOOH}$, the calculation leads to the folloving filling scheme (several last orbitals from 15 filled are given only and the notation as in Fig. 5 was used):

$$
\begin{gathered}
\ldots \mathrm{O}^{11}\left(2 p_{x}\right)^{2} \mathrm{O}^{11}\left(2 p_{z}\right)^{2} \mathrm{O}^{11}\left(2 p_{y}\right)^{2} \mathrm{O}^{12}\left(2 p_{z}\right)^{2} \mathrm{O}^{12}\left(2 p_{y}\right)^{2} \mathrm{O}^{12}\left(2 p_{x}\right)^{2} \\
\mathrm{C}^{11}\left(2 p_{y}\right)^{0} \mathrm{~N}(2 s)^{0} \mathrm{C}^{12}(2 s)^{0} \mathrm{H}^{16}(1 s)^{0} \ldots .
\end{gathered}
$$

It may be noticed that the highest filled MO of the ground state are formed by the same oxygen atomic orbitals as in $\mathrm{HCOOH}$ and $\mathrm{HCOO}^{-}$ions and only the order of the oxygens orbitals $\left(2 p_{z}\right)$ and $\left(2 p_{y}\right)$ is reversed. The unfilled orbitals are changed as a consequence of different bonding of $\mathrm{C}^{11}$ carbon atom but the first unfilled orbitals are composed mainly of $\mathrm{C}^{11}\left(2 p_{y}\right) \mathrm{AO}$ which is antibonding and some analogy to filling scheme (2) can be found. Within the CI treatment procedure as above it was found for the lowest singlet electronic transition for $\mathrm{G}^{+}$ of Gly I of TGS crystal (i.e. $E=5.56 \mathrm{eV}$ - see Table I) that the overlapping of several electron configurations is much stronger than in the case of HCOOH. It was found that the electron excitation from $15 \mathrm{MO}$ to $16 \mathrm{MO}$ (i.e. from the highest filled orbital to the first unfilled orbital in scheme (3)) is contributing in $50 \%$, the excitation from 15 to $17 \mathrm{MO}$ is contributing in $20 \%$, the excitation contributed by $5 \%$ is from 13 to 16 orbital and the rest is from other types of excitations. So if the main $\mathrm{AO}$ and stronger excitation are taken into account only the lowest singlet electronic transition may be written as: $\mathrm{O}^{12}\left(2 p_{x}\right) \rightarrow \mathrm{C}^{11}\left(2 p_{y}\right)$ and in analogy to $\mathrm{HCOOH}$, the nature of that transition may be still regarded as of the $n-\pi^{*}$ type. In other words this transition is not completely localised within the $\mathrm{C}^{11} \mathrm{O}^{12}$ double bond as in the archetype carbonyl chromophore of formaldehyde but involves a more complicated charge transfer from both oxygen atoms throughout the carbons atoms to nitrogen atom. This process involves several more AO of both oxygen $\left(\mathrm{O}^{11}\left(2 p_{z}\right), \mathrm{O}^{12}\left(2 p_{y}\right)\right)$ and carbon $\left(\mathrm{C}^{11}\left(2 p_{y}\right), \mathrm{C}^{12}\left(2 p_{x}\right)\right)$ atoms as well as nitrogen $(\mathrm{N}(2 s))$ and hydrogen $\left(\mathrm{H}^{11}(1 s)\right)$ together with the main ones mentioned above. 
It is worth noticing that an identical procedure performed in the calculation for $\mathrm{G}^{+}$of Gly II of TGS crystal and DGSe crystal as well as for Ala molecule, give similar - in comparison to $\mathrm{G}^{+}$of Gly I - information about a type of the lowest electronic transition and $\mathrm{AO}$ involved.

The results of the calculations of the electronic excitation energies are given in Tables I and II, along with the oscillator power $f$, the parameter describing intensity of a given band. High $f$ refers to the allowed electronic transitions, small $f$ (of the order of $10^{-3}$ ) means that the transition is forbidden and the corresponding

TABLE I

Calculated excitation energies $E$ (in $[\mathrm{eV}]$ ), and the respective oscillator strengths $f$ for glycine molecule and ion assuming their crystal geometry (crystallographic data after the paper [36] for TGS and [37] for DGSe).

\begin{tabular}{c|c|r|r|r|r|r|r}
\hline \hline \multicolumn{4}{c|}{ Gly I } & \multicolumn{2}{c|}{ Gly II } & \multicolumn{2}{c}{ Gly $^{a}$} \\
\hline \multicolumn{2}{c|}{ G } & \multicolumn{2}{c|}{$\mathrm{G}^{+}$} & \multicolumn{2}{c}{$\mathrm{G}^{+}$} & \multicolumn{2}{c}{$\mathrm{G}^{+}$} \\
\hline$E$ & $f$ & \multicolumn{1}{c|}{$E$} & \multicolumn{1}{c}{$f$} & \multicolumn{1}{c}{$E$} & $f$ & $E$ & $f$ \\
\hline 5.06 & 0.020 & 5.56 & 0.001 & 5.69 & 0.001 & 5.86 & 0.002 \\
5.93 & 0.002 & 7.85 & 0.135 & 7.70 & 0.186 & 7.96 & 0.152 \\
6.63 & 0.007 & 8.31 & 0.040 & 8.21 & 0.005 & 8.15 & 0.025 \\
7.36 & 0.009 & 8.99 & 0.058 & 8.84 & 0.038 & 8.33 & 0.029 \\
8.01 & 0.027 & 9.70 & 0.011 & 9.20 & 0.118 & 9.02 & 0.078 \\
8.04 & 0.049 & 9.82 & 0.022 & 9.50 & 0.020 & 9.26 & 0.027 \\
8.48 & 0.083 & 9.98 & 0.052 & 10.04 & 0.004 & 9.75 & 0.007 \\
9.00 & 0.056 & 10.18 & 0.101 & 10.40 & 0.002 & 10.28 & 0.026 \\
\hline
\end{tabular}

\section{TABLE II}

Comparison of the calculated excitation energies $E$ (in $[\mathrm{eV}]$ ) and respective oscillator strengths $f$ for $\mathrm{Ala}$, Gly $\mathrm{I}$, and $\mathrm{HCOOH}$ molecules (crystallographic data after: [39] (Ala), [36] (Gly I), [38] ( $\mathrm{HCOOH})$ ).

\begin{tabular}{c|c|c|c|c|c}
\hline \hline \multicolumn{2}{c|}{ Ala } & \multicolumn{2}{c|}{ Gly } & \multicolumn{2}{c}{ HCOOH } \\
\hline$E$ & $f$ & $E$ & $f$ & $E$ & $f$ \\
\hline 5.41 & 0.003 & 5.06 & 0.020 & 5.13 & 0.002 \\
5.69 & 0.002 & 5.93 & 0.002 & 7.64 & 0.153 \\
5.97 & 0.001 & 6.63 & 0.007 & 7.86 & 0.000 \\
6.81 & 0.019 & 7.36 & 0.009 & 8.41 & 0.087 \\
6.90 & 0.004 & 8.01 & 0.027 & 9.30 & 0.011
\end{tabular}


band appears because of the violation of the selection rules. The results obtained for the first eight electronic excitation energies (Table I) are more or less similar for an individual glycine ion belonging to different crystals. Much greater differences were found between the excitation energies calculated for the Gly I ion and Gly I molecule of the same geometry (Table I). In the crystals we obviously deal with a glycine ion, so this results should be used for comparison with experiment (Fig. 2). The energy of the first electronic excitation for the glycine ion, independently of which crystal it comes from, ranges between 5.6 and $5.9 \mathrm{eV}$, and its oscillator power is small $\left(f \approx 1 \times 10^{-3}\right.$ ) (Table $\mathrm{I}$ ). These values are in very good agreement with the first weak band observed for all crystals studied and their aqueous solutions $(\sim 6 \mathrm{eV})$ (Figs. 2 and 3 ). A small value of $f$ of the first electronic transition suggests a dominant role of the symmetry of carboxyl chromophore.

The above discussed experimental results of DR and optical absorption spectra by its similarity and together with the information from calculations of the ground state and electronic excitation energy indicate that the absorption band at about $6 \mathrm{eV}$ in the spectra of TGS-type crystals can be temporarily assigned to the $n-\pi^{*}$ transition in carboxyl chromophore of glycine.

The optical activity known only in the visible region [4] and the optical absorption of the glycine crystals observed in the spectral range considered are definitely affected by the electronic transitions below $\sim 7.5 \mathrm{eV}$ (see Tables I, II, and Fig. 2), characterised by a high oscillator power $f(0.04-0.1)$. The electronic transitions in this range of energies can be also localised in the carboxyl group [30-32] or in the anions $\mathrm{XO}_{4}^{2-}[42-44]$.

\section{Conclusions}

A weak absorption band showing no optical activity has been recorded at about $206 \mathrm{~nm}$ for aqueous solutions of glycine crystals, see Fig. 3. For glycine single crystals a weak band has also been observed at about $208 \mathrm{~nm}$ (Fig. 2), which is strongly overlapped by the bands corresponding to the electronic transitions in the more short-wavelength range (below $7.5 \mathrm{eV}$ ). The optical activity of this particular band $(208 \mathrm{~nm}$ ) has not been directly observed in the crystals but it cannot be excluded. The position and intensity of the band at $\lambda \approx 206 / 208 \mathrm{~nm}$ in the spectra of glycine crystals and their aqueous solutions are in good agreement with the results of the electronic excitation energy calculated for the glycine ion assuming its crystal geometry. This electronic transition, revealed also in the spectrum of the oblique reflection [15] has been temporarily assigned to the $n-\pi^{*}$ type in the carboxyl chromophore of glycine.

\section{Acknowledgments}

The author wishes to thank Prof. J. Stankowska and Dr A. Czarnecka for providing some of the single crystals used in the studies and to Prof. J. Lipiński 
for providing the computer program and discussion. I would like to acknowledge the Referee for his stimulated comments.

\section{References}

[1] Landolt-Börnstein, Numerical Data and Functional Relationships in Science and Technology, New Series, Group III, Vol. 3, Springer-Verlag, Berlin 1969; Group III, Vol. 9, Springer-Verlag, Berlin 1975.

[2] M.S. Cedrik, Fiziczeskije swojstwa kristałtow semejstwa triglicinsulfata w zawisimosti ot usowij wyraszcziwanija (Physical Properties of Triglycine Sulphate Crystals Family Grown in Different Conditions), Nauka i Technika, Minsk 1986 (in Russian).

[3] R.B. Lal, A.K. Batra, Ferroelectrics 142, 51 (1993).

[4] M. Koralewski, Optical Activity and its Relation with the Ferroelectric State of Selected Frroelectric Crystals, Ser. Phys. 73, A. Mickiewicz University Press, Poznań 2000 (in Polish).

[5] W. Kaminski, A.M. Glazer, Phase Transitons 66, 1 (1998).

[6] J. Przesławski, Acta Phys. Pol. A 95, 997 (1999).

[7] B. Andrievskii, O. Myskchyskyn, M. Romanyuk, Phys. Status Solidi B 203, 549 (1997).

[8] G. Montemezzani, J. Fousek, P. Günter, J. Stankowska, Appl. Phys. Lett. 56, 2367 (1990).

[9] K.A. Verkhovskaya, V.M. Fridkin, Fiz. Tverd. Tela 8, 3129 (1966).

[10] B. Hilczer, S. Balanicka, Phys. Status Solidi A 19, 717 (1973).

[11] A.K. Abass, F.Y.M. Al-Eithan, R.H. Misho, Phys. Status Solidi A 89, 225 (1985).

[12] N.A. Romanyuk, I.I. Polovinko, I.F. Viblyj, Optika i Spektr. 43, 793 (1977).

[13] L.M. Guseva, A.S. Sonin, Fiz. Tverd. Tela 10, 925 (1968).

[14] A. Abu El-Fadl, Cryst. Res. Technology 34, 1047 (1999).

[15] N.A. Romanyuk, B.V. Andrzyevskij, I.S. Zheludev, Ferroelectrics 21, 333 (1978).

[16] J. Stankowska, J. Polovinko, N. Romanyuk, Acta Phys. Pol. A 55, 803 (1979).

[17] J. Mróz, S. Gębala, Optica Applicata XII, 401 (1982).

[18] V. Winterfeldt, G. Schaack, A. Klöpperpieper, Ferroelectrics 15, 21 (1971).

[19] T.R. Griffiths, Anal. Chem. 35, 1077 (1963).

[20] P. Kubelka, F. Munk, Z. Techn. Physik 12, 593 (1931).

[21] G. Kortüm, Reflexionspektroskopie, Springer-Verlag, New York 1969.

[22] M. Szafrański, M. Koralewski, Acta Phys. Pol. A 75, 705 (1989).

[23] J. Lipiński, S. Styrcz, GRINDOL Program, Technical University of Wrocław, Wrocław 1988, Poland.

[24] J. Lipiński, A. Nowek, H. Chojnacki, Acta Phys. Pol. A 53, 229 (1978).

[25] J. Lipiński, J. Leszczyński, Theor. Chim. Acta (Berl.) 63, 305 (1983).

[26] J. Stankowski, Phys. Rep. 77, 1 (1981). 
[27] L.D. Kislovski, E.K. Galanov, L.A. Shuvalov, Optika i Spektr. 24, 100 (1968).

[28] D. Chakraborty, S. Manogaran, Chem. Phys. Lett. 294, 56 (1998) and references therein.

[29] A. Fernandez-Ramos, Z. Smedarchina, W. Siebrand, M.Z. Zgierski, J. Chem. Phys. 113, 9714 (2000) and references therein.

[30] H.M. Jaffé, M. Orchin, Theory and Application of Ultraviolet Spectroscopy, Wiley, New York 1962

[31] J.P. Simons, Photochemistry and Spectroscopy, PWN, Warszawa 1976 (in Polish).

[32] J.M. Sidman, Chem. Rev. 58, 689 (1958).

[33] P. Crabbe, An Introduction to the Chiroptical Methods in Chemistry, PWN, Warszawa 1974 (in Polish).

[34] C. Djerassi, Optical Rotatory Dispersion, Mc Graw-Hill, New York 1960.

[35] F. Richardson, R.W. Strickland, Tetrahedron 31, 2309 (1975).

[36] S.R. Fletcher, E.T. Keve, C. Skapski, Ferroelectrics 14, 775 (1976).

[37] S. Olejnik, Ph.D. Thesis, Institute for Low Temperature and Structure Research PAS, Wrocław 1975 (in Polish).

[38] S.D. Peyerimhoff, R.J. Buenker, J. Chem. Phys. 50, 1846 (1969).

[39] H.J. Simpson Jr., R.E. Marsh, Acta Crystallogr. 20, 550 (1966).

[40] L.M. Beaty-Travis, D.C. Moule, H. Liu, E.C. Lim, R.H. Judge, J. Mol. Spectrosc. 205, 232 (2001)

[41] T.L. Ng, S. Bell, J. Mol. Spectrosc. 50, 166 (1974).

[42] J.A. Connor, I.H. Hillier, V.R. Saunders, M. Barber, Mol. Phys. 23, 81 (1972).

[43] I.H. Hillier, V.R. Saunders, Int. J. Quant. Chem. 4, 203 (1970).

[44] W.G. Plechanov, W.S. Osminin, Optika i Spektr. XXXIX, 604 (1975). 